\title{
Guías de Práctica Clínica para el Tratamiento del Tabaquismo, Chile 2017
}

\author{
MARÍA PAZ CORVALÁN B. ${ }^{1}$, LEONARDO VÉJAR M. ${ }^{2}$, CLAUDIA BAMBS S. ${ }^{3}$, JUANA PAVIÉ G. ${ }^{4}$, MÓNI- \\ CA ZAGOLIN B. ${ }^{5}$ y JAIME CERDA L. ${ }^{6}$ \\ EN REPRESENTACIÓN DEL GRUPO FÉNIX (GRUPO FORMADO POR ESPECIALISTAS NACIONALES \\ INTEGRADOS PRO CONTROL DEL TABACO EN SIGLO XXI)*
}

\section{Clinical Practice Guidelines for Smoking Cessation}

Considering that 40\% (ENS 2010) of the Chilean general population smokes, the Chilean Society of Respiratory Diseases in collaboration with the Chilean Societies of Cardiology and, Endocrinology and Diabetes, formed an interdisciplinary group, who issued a set of recommendations for the treatment of the smoker, methodologically advised by experts. These interventions should be prioritized in highrisk groups. Methods: The panel elaborated and graded the recommendations following the GRADE methodology. To assess the effect of each intervention, systematic reviews and randomized clinical trials were identified. In addition, a search of studies done with the Chilean population was carried out. For each of the questions, the panel determined the direction and strength of the recommendation through a decision evidence table. Recommendations: For all smokers, the panel recommends using brief counseling ABC on non-intervention, using mobile telephone interventions on non-intervention, using text message on non-intervention, (strong recommendation; moderate certainty in the evidence of the effects). For motivated individuals, with indication for quitting drugs the panel recommends using nicotine replacement therapy on non-intervention, using bupropion on non-intervention, using varenicline on non-intervention. (strong recommendation; moderate certainty in the evidence of the effects). Discussion: This clinical practice guide provides recommendations based on the evidence for smoking cessation.

Key words: Smoking cessation; tobacco use disorder; varenicline; burpopion; nicotine; counseling.

1 Programa de Tabaquismo Centro Médico Fundación del Banco Estado, Santiago, Chile. Coordinadora Comisión Tabaco, Sociedad Chilena Enfermedades Respiratorias, Chile.

2 Leonardo Véjar M. Programa Tabaquismo Servicio de Salud Metropolitano Sur Oriente, Santiago, Chile.

3 Departamento de Salud Pública y Centro Avanzado de Enfermedades Crónicas ACCDiS (Fondap 15130011). Escuela de Medicina, Pontificia Universidad Católica de Chile.

4 Dpto. Enfermedades Respiratorias, Programa de Tabaquismo, Hospital de Quillota, 5a Región, Chile.

5 Programa de Tabaco, Clínica Santa María, Santiago, Chile. Profesora Asociada, Facultad de Medicina, Universidad de Chile.

6 Dpto. Salud Pública, Facultad de Medicina, Pontificia Universidad Católica de Chile.

* Integran además el Grupo Fénix: Marisol Acuña A., Mónica Acuña M., Rodrigo Alonso K., Juan Alcántara C., Francisco Arancibia H., Sergio Bello S., Ángel Benítez V., Mauricio Burotto P., Alex Campos A., Ada Cuevas M., Haydée Chamorro D., Manuel De la Prida C., Fernando Descalzi M., Marcia Erazo B., Maria José Escaffi F., Mauricio Fernández R., Juan Guillermo Gormaz A., Ana María Herrera G., Jaime Leppe Z., Philippa Moore C., Pedro Peña G., Francisca Rodríguez C., Jaime Sapaj M., Daniel Seijas B., Néstor Soto I., Lisandro Stuardo T., Juan Trebilcock G., Gonzalo Valdivia C., Raúl Villarroel P. y Tania Vizcarra O.

Asesores metodológicos: María Francisca Rodríguez, USS e Ignacio Neumann B., PhD, PUC.

Financiamiento: Los recursos económicos requeridos para la elaboración de esta guía fueron proporcionados por la Sociedad Chilena de Enfermedades Respiratorias, entidad que brindó además el patrocinio para esta iniciativa.

Conflictos de interés: Los participantes en la elaboración de esta guía declaran no tener conflictos de interés.

NOTA: Por su relevancia nacional estas guías de práctica clínica para el tratamiento del tabaquismo serán publicadas simultáneamente en la Revista Chilena de Enfermedades Respiratorias y en la Revista Médica de Chile. Los archivos complementarios conteniendo los apéndices 1 a 7 citados en este texto pueden ser consultados en: www.scielo.cl, vol 33 , n 3 . 


\section{RESUMEN}

Considerando la prevalencia de 40\% (ENS 2010) de consumo de tabaco en Chile la Sociedad Chilena de Enfermedades Respiratorias en colaboración con las Sociedades Chilenas de Cardiología; Endocrinología y Diabetes formó un grupo interdisciplinario que emitió un conjunto de recomendaciones para el enfrentamiento del paciente fumador, asesorado metodológicamente por expertos. Estas intervenciones deben priorizarse en grupos de alto riesgo. Métodos: El panel elaboró y graduó las recomendaciones siguiendo la metodología GRADE. Para estimar el efecto de cada intervención, se identificó revisiones sistemáticas y estudios clínicos aleatorizados. Además, se realizó una búsqueda de estudios realizados con población chilena. Para cada una de las preguntas, el panel determinó la dirección y fuerza de la recomendación mediante una tabla de la Evidencia a la Decisión. Recomendaciones: Para todos los fumadores, el panel recomienda usar consejería breve sobre no intervención, consejería vía telefonía móvil sobre no intervención, y mensajes de texto sobre no intervención (recomendación fuerte; certeza moderada en la evidencia de los efectos). Para los individuos motivados, con indicación de fármacos para dejar de fumar el panel recomienda terapia de reemplazo de nicotina sobre no intervención, bupropión sobre no intervención, vareniclina sobre no intervención (recomendación fuerte; certeza moderada en la evidencia de los efectos). Discusión: Se emiten recomendaciones basadas en la evidencia para el tratamiento del tabaquismo.

Palabras clave: Tabaquismo; dejar de fumar; vareniclina; bupropión; nicotina; tabaquismo; consejería.

\section{Introducción}

Las Enfermedades Crónicas no Transmisibles como cáncer, enfermedades cardiovasculares, enfermedades respiratorias y diabetes son la epidemia del siglo XXI. El consumo de tabaco es un factor de riesgo común a todas ellas. Chile tiene una historia de alta prevalencia de consumo de tabaco en escolares, adolescentes y adultos, al igual que en mujeres embarazadas. Ello produce una elevada carga de enfermedad y mortalidad atribuible al consumo de tabaco, y crecientes e importantes costos económicos derivados del tratamiento de enfermedades relacionadas ${ }^{1-6}$. Más de 1 billón de pesos es gastado en Chile cada año para tratar los problemas de salud provocados por el tabaco 5 .

El tabaquismo se considera una enfermedad crónica y una adicción compleja, con componentes físicos, psicológicos y sociales. La nicotina, compuesto orgánico presente en el tabaco, es una de las sustancias más adictivas que existe junto con la cocaína y la heroína ${ }^{7}$.

En Chile, al momento de confeccionar esta Guía de Práctica Clínica (GPC), existen diversas intervenciones para ayudar al cese del tabaquismo; tanto de tipo no-farmacológico (cognitivo conductuales, entrevista motivacional) entre ellas consejería breve, terapia intensiva, líneas telefónicas (Salud Responde MINSAL 6003607777, este número esta en los envases de cigarrillos), apps, mensajes de texto, sitios $w e b^{8}$; como far- macológicas, es decir terapia de reemplazo nicotínico, bupropión, vareniclina y otras de segunda línea. Existen terapias no respaldadas por la evidencia en la actualidad como cigarrillo electrónico, láser, hipnosis, flores de Bach y acupuntura.

\section{Propósito de la GPC, audiencia y población objetivo:}

El propósito de esta guía es presentar recomendaciones basadas en evidencia sobre las intervenciones disponibles para dejar de fumar. $\mathrm{Su}$ audiencia objetivo corresponde a todos los profesionales de la salud y su población objetivo corresponde a personas fumadoras atendidas en ambientes ambulatorios u hospitalarios, además de poblaciones especiales como embarazadas, adolescentes y pacientes con enfermedad psiquiátrica (compensada por al menos tres meses). Nos parece que se debe focalizar en pacientes de alto riesgo diabéticos, cardiovasculares, respiratorios y con cáncer, tanto en el ambiente ambulatorio como hospitalario.

\section{Métodos}

El panel elaboró y graduó las recomendaciones siguiendo la metodología GRADE, de acuerdo a los estándares propuestos por el Institute of Medicine y la Guidelines International Network ${ }^{9}$. 
Tabla 1. Preguntas priorizadas para la guía

\begin{tabular}{|c|c|c|c|}
\hline Preguntas & Población & Intervención & Comparación \\
\hline $\begin{array}{l}\text { P1. ¿Se debe utilizar con- } \\
\text { sejería breve versus no } \\
\text { intervención en fumadores? }\end{array}$ & Fumadores & Consejería Breve & Nada \\
\hline $\begin{array}{l}\text { P2. ¿Se deben utilizar in- } \\
\text { tervenciones basadas en } \\
\text { telefonía móvil versus no } \\
\text { intervención en fumadores? }\end{array}$ & Fumadores & $\begin{array}{l}\text { Intervenciones basadas en } \\
\text { telefonía móvil }\end{array}$ & Nada \\
\hline $\begin{array}{l}\text { P3. ¿Se deben utilizar in- } \\
\text { tervenciones basadas en } \\
\text { mensajes de texto versus no } \\
\text { intervención en fumadores? }\end{array}$ & Fumadores & $\begin{array}{l}\text { Intervenciones basadas en } \\
\text { mensajes de texto }\end{array}$ & Nada \\
\hline $\begin{array}{l}\text { P4. ¿Se debe usar terapia } \\
\text { de reemplazo nicotínico } \\
\text { sobre no intervención en } \\
\text { individuos dependientes de } \\
\text { nicotina con intención de } \\
\text { dejar de fumar? }\end{array}$ & Fumadores & $\begin{array}{l}\text { Terapia de reemplazo ni- } \\
\text { cotínico }\end{array}$ & Nada \\
\hline $\begin{array}{l}\text { P5. ¿Se debe usar bupro- } \\
\text { pión sobre no intervención } \\
\text { en individuos dependientes } \\
\text { de nicotina con intención de } \\
\text { dejar de fumar? }\end{array}$ & Fumadores & Bupropión & Nada \\
\hline $\begin{array}{l}\text { P6. ¿Se debe usar vareni- } \\
\text { clina sobre no intervención } \\
\text { en individuos dependientes } \\
\text { de nicotina con intención de } \\
\text { dejar de fumar? }\end{array}$ & Fumadores & Vareniclina & Nada \\
\hline
\end{tabular}

\section{Composición del Panel}

Mediante invitaciones personales fueron convocados todos los profesionales de la salud de alguna manera involucrados en programas control del tabaco a nivel nacional.

\section{Formulación de preguntas y selección de des- enlaces}

El panel elaboró preguntas definiendo explícitamente la población de interés, la intervención y la comparación. Luego de un proceso de priorización, se seleccionaron seis preguntas (Tabla 1).

Para cada una de las preguntas se seleccionaron los desenlaces de interés siguiendo un proceso estandarizado: primero, el panel identificó todos los posibles desenlaces atingentes, para luego evaluar su importancia relativa para la decisión clínica.

\section{Revisión de la evidencia respecto de los efectos de las intervenciones}

Para estimar el efecto relativo de cada intervención, primero se identificó revisiones sistemáticas pertinentes mediante una búsqueda en la biblioteca de la Colaboración Cochrane y en MEDLINE. Luego se realizó una búsqueda de estudios randomizados publicados luego de la fecha de búsqueda de las revisiones sistemáticas encontradas en MEDLINE, EMBASE y CENTRAL (el apéndice 1 describe la estrategia de búsqueda en detalle, link en la nube). Cuando no se identificó algún estudio randomizado no contenido en las revisiones sistemáticas, se utilizó los resultados del meta-análisis original sin cambios. Cuando se identificó uno o más estudios randomizados no contenidos en una revisión sistemática, se actualizó el meta-análisis usando el programa RevMan 5.3 (Version 5.3 Copenhagen: The Nordic Cochrane Centre, The Cochrane Collaboration, 2014) mediante el método de Mantel-Haenszel y el modelo de efectos aleatorios.

Además, para obtener una estimación del riesgo basal de los distintos desenlaces, se realizó una búsqueda electrónica en MEDLINE y EMBASE de estudios observacionales realizados con población chilena (apéndice 1).

Los efectos absolutos de las intervenciones fueron calculados a partir de los riesgos basales identificados en la población chilena y el riesgo relativo obtenido del meta-analisis de los estudios 
randomizados relevantes.

Para cada desenlace seleccionado, se evaluó la certeza en la evidencia siguiendo la metodología GRADE ${ }^{10}$. Dos investigadores juzgaron el riesgo de sesgo del conjunto de evidencia, la inconsistencia entre los resultados de los estudios individuales, la precisión de los estimadores combinados en el meta-análisis, la aplicabilidad de la evidencia encontrada a la pregunta y la probabilidad de sesgo de publicación. En el caso de los estudios randomizados incluidos en revisiones sistemáticas se aceptó el juicio de riesgo de sesgo para los estudios individuales de los autores de la revisión. Para los estudios randomizados no incluidos en revisiones sistemáticas, se evaluó el riesgo de sesgo a nivel de cada desenlace siguiendo los principios de la Cochrane Risk of Bias Tool ${ }^{11}$.

Finalmente, los efectos relativos y absolutos de las intervenciones, así como la evaluación de la certeza de la evidencia se presentaron al panel en una tabla de resumen de evidencia.

\section{Revisión de la evidencia respecto de los valores y preferencias}

Para identificar estudios describiendo la importancia relativa de los distintos desenlaces seleccionados, así como de la valoración de los pacientes de las distintas intervenciones, se condujo una búsqueda de estudios observacionales en MEDLINE y EMBASE (apéndice 1).

\section{Determinación de la dirección y fuerza de la recomendación}

Para cada una de las preguntas, el panel determinó en consenso la dirección y fuerza de la recomendación siguiendo la metodología GRADE. Para ello se elaboró una tabla de la Evidencia a la Decisión utilizando el programa GRADEpro Guideline Development Tool (McMaster GRADE Center, Hamilton, Ontario, Canadá, 2016). Cada tabla resume la evidencia disponible respecto de los efectos de la intervención, la certeza en la evidencia, los valores y preferencias de los pacientes, así como información respecto a la accesibilidad, aceptabilidad e impacto en la equidad de cada intervención. El panel decidió no incluir evidencia de costos en la determinación de la dirección y fuerza de la recomendación.

\section{Como utilizar esta guía}

Las recomendaciones fueron clasificadas como "fuerte" o "condicional". La Tabla 2 muestra la interpretación correcta de los distintos grados de recomendación.

\section{Recomendaciones}

1. ¿Se debe utilizar la consejería breve versus no intervención en fumadores para la cesación del consumo de tabaco?

Recomendación 1: Para todos los fumadores, el panel recomienda usar consejería

Tabla 2. Interpretación de recomendaciones fuertes y condicionales

\begin{tabular}{|c|c|c|}
\hline & Recomendación Fuerte & Recomendación Condicional \\
\hline Pacientes & $\begin{array}{l}\text { Todos o casi todos los individuos } \\
\text { adecuadamente informados elegirían } \\
\text { la intervención propuesta }\end{array}$ & $\begin{array}{l}\text { La mayoría de los individuos ade- } \\
\text { cuadamente informados elegirían la } \\
\text { intervención propuesta, sin embargo, } \\
\text { una proporción importante podría } \\
\text { decidir lo contrario }\end{array}$ \\
\hline Clínicos & $\begin{array}{l}\text { Todos o casi todos los individuos } \\
\text { debieran recibir la intervención } \\
\text { propuesta. Las decisiones de ayuda } \\
\text { suelen ser de escasa utilidad en este } \\
\text { contexto. El foco del clínico debiera } \\
\text { estar en superar las barreras para la } \\
\text { implementación de la intervención } \\
\text { propuesta }\end{array}$ & $\begin{array}{l}\text { Es importante reconocer que eleccio- } \\
\text { nes diferentes pueden ser apropiadas } \\
\text { para individuos distintos. El foco del } \\
\text { clínico debiera estar en ayudar a cada } \\
\text { paciente a tomar decisiones concor- } \\
\text { dantes con sus valores y preferencias. } \\
\text { Las decisiones de ayuda suelen ser } \\
\text { muy útiles en este contexto }\end{array}$ \\
\hline Políticas de Salud & $\begin{array}{l}\text { La recomendación puede ser adoptada } \\
\text { como política de salud en todos o casi } \\
\text { todos los casos. } \\
\text { Este tipo de recomendaciones son un } \\
\text { buen sustrato para elaborar medidas } \\
\text { de desempeño }\end{array}$ & $\begin{array}{l}\text { La elaboración de una política de } \\
\text { salud requeriría de un debate extenso } \\
\text { con los distintos actores relevantes }\end{array}$ \\
\hline
\end{tabular}


breve sobre no intervención (recomendación fuerte; certeza moderada en la evidencia de los efectos).

\section{Resumen de la evidencia}

Identificamos una revisión sistemática $(\mathrm{RS})^{12}$ y un estudio clínico aleatorizado (ECA) ${ }^{13}$ adicional (en el apéndice 2, se encuentra la tabla de resumen de hallazgos (SoF) y la tabla de la evidencia a la decisión $[\mathrm{EtD}])$.

\section{Beneficios}

Por cada 100 pacientes en que se efectúa la consejería breve, 5 más dejan de fumar en comparación a cuando no se realiza (certeza moderada).

\section{Riesgos}

No se identificó riesgos en relación con el uso de la consejería breve.

\section{Justificación de la recomendación}

El panel consideró que, si bien no existe evidencia respecto del efecto de la consejería breve en el pronóstico de los fumadores, el beneficio en dejar de fumar justifica una recomendación fuerte. Además, el panel consideró que la consejería breve es una intervención factible de implementar y que resulta aceptable por pacientes y prestadores.

\section{Consideraciones de implementación ABC-D:}

La consejería breve consiste en realizar el ABC-D y registrar en la ficha clínica ${ }^{14}$. A: significa averiguar si el paciente fuma o no (debe hacerse en todo consultante independiente del motivo de consulta); B: implica dar un breve consejo firme, personalizado y repetido cada vez que un fumador consulte (en general los pacientes requieren varios intentos para dejar de fumar definitivamente), hablar del mal olor de la ropa, del color amarillo de la piel, dientes y uñas, la producción de arrugas, la sequedad del pelo, infertilidad e impotencia y $\mathbf{C}$ : cesación del tabaquismo que quiere decir (I) Referir a consejería telefónica (MINSAL Salud Responde, 6003607777, este número está escrito en los envases de cigarrillos), a un sitio $w e b^{8}$, a un centro que tenga implementado tratamiento de cesación del tabaquismo, o (II) Tratar, que implica (1) preguntar al paciente si quiere dejar de fumar de aquí a un mes más, (sólo si la respuesta es positiva existe la motivación para tratamiento) (2) saber cuántos cigarrillos fuma y a qué hora fuma su primer cigarrillo, porque si fuma más de 10 cigarrillos al día o si fuma su primer cigarrillo durante la primera media hora desde que se levanta el paciente requiere fármacos (3) Uso de fármacos (a) en los pacientes con antecedentes o sospecha de patología psiquiátrica inestable se debe pedir autorización de equipo de Salud Mental o al psiquiatra para iniciar un tratamiento farmacológico. Las Guías de Práctica Clínica de Tratamiento de Personas con Depresión del MINSAL ${ }^{15}$, presentan tres preguntas que pueden ayudar: ¿Se ha sentido cansado o decaído casi todos los días?, ¿Se ha sentido triste, deprimido o pesimista casi todos los días? ¿Siente que ya no disfruta o ha perdido en el interés por cosas $o$ actividades que antes le resultaban agradables o entretenidas? La patología psiquiátrica estable por tres meses permite el uso seguro de TRN, bupropión y vareniclina ${ }^{16}$ (b) preguntar por antecedente de convulsiones o traumatismo encéfalo craneano, uso concomitante de nortriptilina o amitriptilina; en estas condiciones está contraindicado el uso de bupropión (4) establecer la fecha para dejar de fumar o "Día D", (primer día sin fumar), que debe ser máximo hasta un mes más desde la primera consulta, e indicar al paciente que empiece a postergar 10 minutos cada cigarrillo y haga algo que o distraiga (cambiar de actividad, consumiendo, chicles, líquidos, frutas, verduras) (5) Prescribir medicamentos, si esto beneficia al paciente. (6) Seguimiento a la semana para ver tolerancia al fármaco, (ya sea personal o telefónico) y controlar una vez a la semana en 3 ocasiones más ${ }^{14}$, los mayores riesgos de recaída son durante el primer mes ${ }^{14}$. Por último, D implica derivar siempre, si es que no dispone de tiempo suficiente para realizar el $\mathrm{ABC}$ completo, ya que incluso a los fumadores ocasionales les produce daño el cigarrillo.

2. ¿Se deben utilizar intervenciones basadas en telefonía móvil versus no intervención en fumadores?

Recomendación 2: Para todos los fumadores el panel recomienda usar intervenciones de telefonía móvil sobre no intervención (recomendación fuerte; certeza moderada en la evidencia de los efectos).

\section{Resumen de la evidencia}

Identificamos una $\mathrm{RS}^{17}$ y ningún ECA adicional (apéndice 3).

\section{Beneficios}

Por cada 100 pacientes en que se utiliza intervenciones de telefonía móvil, 5 más dejan de fumar en comparación a cuando no se realiza (certeza moderada). 


\section{Riesgos}

No se identificó riesgos en relación al uso de telefonía móvil.

\section{Justificación de la recomendación}

El panel consideró que, si bien no existe evidencia respecto del efecto de las intervenciones de telefonía móvil en el pronóstico de los fumadores, el beneficio en dejar de fumar justifica una recomendación fuerte.

\section{Consideraciones de implementación}

La mHealth, se refiere al uso de dispositivos móviles como una herramienta de telemedicina, ideal para la promoción, prevención y control de enfermedades, facilitando no sólo la comunicación entre el equipo de salud y los pacientes, sino también entre pacientes y con la red de recursos comunitarios $^{18}$.

En Chile, un 91\% de la población tiene acceso a internet y un $80 \%$ de los accesos son a través de dispositivos móviles ${ }^{19}$. Esto convierte a internet y la telefonía móvil en herramientas particularmente atractivas para intervenciones en salud, con el potencial de contribuir a disminuir las brechas socioeconómicas de acceso a tratamiento de apoyo a la cesación tabáquica en nuestra población ${ }^{19,20}$.

3. ¿Se deben utilizar intervenciones basadas en mensajes de texto versus no intervención en fumadores?

Recomendación 3: Para todos los fumadores, el panel recomienda usar mensaje de texto sobre no intervención (recomendación fuerte; certeza moderada en la evidencia de los efectos).

\section{Resumen de la evidencia}

Identificamos una $\mathrm{RS}^{17}$ y ningún ECA adicional (apéndice 4).

\section{Beneficios}

Por cada 100 pacientes en que se utilizan mensajes de texto, 5 más dejan de fumar en comparación a cuando no se realiza (certeza moderada).

\section{Riesgos}

No se identificó riesgos en relación con el uso de mensajes de texto.

\section{Justificación de la recomendación}

El panel consideró que, si bien no existe evidencia respecto del efecto de la mensajería de texto en el pronóstico de los fumadores, el benefi- cio en dejar de fumar justifica una recomendación fuerte.

\section{Consideraciones de implementación}

Los estudios que implican intervenciones a través de mensajes de texto para el apoyo a la cesación tabáquica, no son directamente extrapolables a contextos sociales y culturales distintos a los de origen (la mayoría provienen de países desarrollados y de habla inglesa), motivo por el cual es necesario desarrollar y evaluar intervenciones de este tipo en nuestra población.

4. ¿Se debe usar terapia de reemplazo nicotínico (TRN) sobre no intervención en individuos con indicación de fármacos y con intención de dejar de fumar?

Recomendación 4: Para los individuos con indicación de fármacos e intención de dejar de fumar, el panel recomienda usar terapia de reemplazo sobre no intervención (recomendación fuerte; certeza moderada en la evidencia de los efectos).

\section{Resumen de la evidencia}

Identificamos una $\mathrm{RS}^{21}$ y ningún ECA adicional (apéndice 5).

\section{Beneficios}

Por cada 100 pacientes en que se utiliza terapia de reemplazo nicotínico, 5 más dejan de fumar en comparación a cuando no se realiza (certeza alta).

\section{Riesgos \\ Por cada 100 pacientes en que se utiliza terapia de reemplazo nicotínico, 1 más desarrolla palpi- taciones en comparación a cuando no se realiza (certeza moderada).}

\section{Justificación de la recomendación}

El panel consideró que, si bien no existe evidencia respecto del efecto de la terapia de reemplazo nicotínico a largo plazo, el beneficio en dejar de fumar justifica una recomendación fuerte.

\section{Consideraciones de implementación}

La TRN se puede usar como terapia única, combinar entre sus formas rápida (chicles) o lenta (parches) o asociar (en general chicles) con otras drogas como bupropión o vareniclina. Se sugiere utilizar parche de nicotina o bupropión con una forma de administración rápida de TRN para lograr la mayor eficacia. TRN y bupropión, usados como terapia única son de igual eficacia ${ }^{21}$ Chicles 
y parches requieren una técnica especial de utilización. Presentación: chicles de 2 y 4 mg, parches de 7, 14 y $21 \mathrm{mg}$ de $24 \mathrm{~h}$ de duración, (en la actualidad solo los chicles existen en Chile, los parches estarán disponibles en 2018). Prescripción: TRN se utiliza a partir del "Día D". Se usa entre 5 a 8 unidades de chicles al día SOS, en general la dosis es de $2 \mathrm{mg}$ para manejo del "craving" o urgencia por fumar, (los pacientes tienden a usar menos chicles de lo que necesitan, por lo que es necesario incentivar el uso). Un cigarrillo es igual a $1 \mathrm{mg}$ de nicotina. En caso de los parches, la dosis se titula de acuerdo con el nivel de adicción, el inicio del tratamiento para los que fuman más de 15 cigarrillos/día o prenden su primer cigarrillo dentro de la primera hora del día es con parches de $21 \mathrm{mg}$ /día y se disminuye cada 2 semanas no recomendándose tiempos superiores a las 8 semanas. La TRN está contraindicada en pacientes inestables hemodinámicamente o con arritmias graves.

Según expertos chilenos, se necesita usar TRN en adolescentes ${ }^{22-27}$ en forma de chicles de $2 \mathrm{mg}$ por 1 a 2 meses, en adolescentes adictos a la nicotina si ellos quieren y piensan que este fármaco lo puede ayudar. Si una embarazada expresa un claro deseo de recibir TRN ${ }^{22,27}$, se sugiere (i) discutir con ella los riesgos y beneficios asociados, (ii) utilizarla sólo si falla la cesación con medidas no farmacológicas (terapia cognitiva conductual) y (iii) utilizar el criterio profesional al decidir si ofrecer la prescripción de TRN; iiii) Si bien no existe consenso en el esquema terapéutico a usar en embarazadas, expertos internacionales sugieren lo siguiente:

Partir con chicles de nicotina de $2 \mathrm{mg}$. Si esto no funciona considerar el uso de parches de nicotina de $24 \mathrm{~h}$ de duración que se colocan en la mañana y se retiran en la noche. Si la paciente fuma menos de 10 cigarrillos al día usar 1 parche al día de 7 o $14 \mathrm{mg}$. Si fuma 10 o más cigarrillos al día partir con 1 parche al día de $14 \mathrm{mg}$. Evaluar cada 2 semanas y considerar la disminución progresiva de los parches de 14 a $7 \mathrm{mg}$. Usar por un máximo de 2 meses. Notar que solo se justifica mantener los parches si la paciente se mantiene sin fumar.

Basado en esquema terapéutico usado en The Mayo Clinic Dependence Center, USA y Division of Primary Care, University of Nottingham, England.

En la actualidad los chicles de nicotina existen en Chile pero no estan disponibles en el sistema público .

5. ¿Se debe usar bupropión sobre no intervención en individuos dependientes de nicotina con intención de dejar de fumar?
Recomendación 5: Para todos los fumadores, el panel recomienda usar bupropión sobre no intervención (recomendación fuerte; certeza moderada en la evidencia de los efectos).

\section{Resumen de la evidencia}

Identificamos una $\mathrm{RS}^{28}$ y ningún ECA adicional (apéndice 6).

\section{Beneficios}

Por cada 100 pacientes en que se utiliza bupropión, 5 más dejan de fumar en comparación a cuando no se realiza (certeza moderada).

\section{Riesgos}

Por cada 100 pacientes, no habrá diferencias en cuanto a la cantidad de eventos adversos serios entre el uso de bupropión y no utilizar nada (certeza moderada).

\section{Justificación de la recomendación}

El panel consideró que, si bien no existe evidencia respecto del efecto del bupropión en el pronóstico de los fumadores cardiópatas hospitalizados, el beneficio en dejar de fumar justifica una recomendación fuerte.

\section{Consideraciones de implementación \\ Bupropión:}

La presentación de bupropión es de $150 \mathrm{mg}$ (administración dos veces al día) y 150 y $300 \mathrm{mg}$ (liberación sostenida se puede tomar 1 vez al día). Indicación una semana antes del prefijado "Día D" (primer día sin fumar) en la forma de 150 $\mathrm{mg}$ en la mañana por 3 días, luego $150 \mathrm{mg}$ por 2 veces, última dosis a las 15-16 h o antes, para minimizar insomnio (30-40\%). Su tiempo de uso es de 2 a 3 meses, aunque podría ser mayor. Está contraindicado en pacientes con antecedentes de convulsiones, tumores del sistema nervioso central, traumatismo encéfalo craneano, bulimia, anorexia o trastorno bipolar, también está contraindicado usarlo junto a inhibidores de la MAO. No se administra en embarazo ni lactancia. Puede ser usado en conjunto con inhibidores de recaptación de la serotonina. En Adultos mayores reducir dosis a $150 \mathrm{mg}$ al día, al igual que en pacientes con deficiente tolerancia al fármaco.

6. ¿Se debe usar vareniclina sobre no intervención en individuos dependientes de nicotina con intención de dejar de fumar?

Recomendación 6: Para los individuos con intención de dejar de fumar dependientes de nicotina, el panel recomienda usar vareniclina sobre no intervención (recomendación fuerte; 
certeza moderada en la evidencia de los efectos).

\section{Resumen de la evidencia}

Identificamos una $\mathrm{RS}^{29}$ y ningún ECA adicional (apéndice 7).

\section{Beneficios}

Por cada 100 pacientes en que se utiliza vareniclina sola, 20 más dejan de fumar en comparación a cuando no se utiliza (certeza moderada).

\section{Riesgos}

Por cada 100 pacientes en que se utiliza vareniclina:

- 20 más presentarán náuseas en comparación a cuando no se utiliza (certeza alta).

- 4 más presentarán insomnio en comparación a cuando no se utiliza (certeza alta).

- 2 más presentaran cefalea en comparación a cuando no se utiliza (certeza alta).

- No habrá diferencias en el número de casos de ideación suicida en comparación a cuando no se utiliza (certeza moderada).

\section{Justificación de la recomendación}

El panel consideró que, si bien no existe evidencia respecto del efecto de la vareniclina en el pronóstico de los fumadores, el beneficio en dejar de fumar justifica una recomendación fuerte.

\section{Consideraciones de implementación}

El panel consideró que los pacientes con mayor dependencia a la nicotina son los que más se beneficiarían del uso de vareniclina. En su inicio, una semana antes del "Día D" se usa una tableta de $0.5 \mathrm{mg}$ en la mañana por 3 días y luego 1 cada 12 del blíster de inicio y luego de una semana se pasa al blíster de mantención, cuyas tabletas son de $1 \mathrm{mg}$ y se usan cada $12 \mathrm{~h}$ con desayuno y cena por 2-3 meses o incluso más prolongado. Es el medicamento de primera línea más efectivo, es seguro, con pocos efectos colaterales (náuseas, que aparecen inicialmente y luego se atenúan), se usa en pacientes con clearance de creatinina mayor a $30 \mathrm{ml} / \mathrm{min}$, no se puede usar en embarazo, lactancia ni adolescentes menores de 18 años se puede reducir dosis a la mitad en pacientes con deficiente tolerancia al fármaco. Este medicamento se encuentra en Chile, pero no está disponible en el sistema público en la actualidad.

\section{Discusión}

La presente Guía de Práctica Clínica para el
Tratamiento del Tabaquismo en Chile (2017) ofrece recomendaciones elaboradas por un grupo multidisciplinario de expertos, basándose su confección en la mejor evidencia disponible. $\mathrm{Su}$ enfoque ha sido en intervenciones farmacológicas y no-farmacológicas para la cesación del tabaquismo. Se espera que la implementación de estas recomendaciones contribuya a reducir la prevalencia de consumo de tabaco en Chile, siendo complementarias a las medidas legales (ej. aumento de impuestos al tabaco, espacios cerrados de uso público libres de humo de tabaco, control de la publicidad) que han sido instauradas progresivamente en nuestro país.

\section{Agradecimientos}

A Carlos Jiménez R y Gustavo Zabert V por la asesoría internacional en la realización de las primeras Guías de Práctica Clínica de Tratamiento del Tabaquismo, Chile 2017. A Gonzalo Valdivia C. y Sergio Bello S. por su apoyo permanente a la gestión de este proyecto, a Taylor Hays, Robert West y Tim Coleman por su consejo en el uso de TRN en embarazadas, a Stan Shatenstein por su ayuda en búsquedas bibliográficas, a José Luis Corvalán M. y Carmen Barros F. por su colaboración en la edición de estas Guías.

\section{Bibliografía}

1.- MINSAL. Encuesta Nacional de Salud de Chile. 2010.

2.- TELGIE R. Prevalencia del fumar en las gestantes controladas en los consultorios municipalizados de la comuna de Quinta Normal (Región Metropolitana, Chile). Cuad Méd Soc (Chile). 2007; 47: 253-7.

3.- MALLOL J, BRANDENBURG D, MADRID R, SEMPERTEGUI F, RAMÍREZ L, JORQUERA D. Prevalencia de tabaquismo durante el embarazo en mujeres chilenas de bajo nivel socioeconómico. Rev Chil Enferm Respir. 2007; 23: 17-22.

4.- SENDA. Décimo Estudio Nacional de Drogas en Población Escolar. Julio, 2014.

5.- PICHÓN RIVIERE A BA, CAPORALE J, ALCARAZ A, AUGUSTOVSKi F, CACCAVO F, VALLEJOS V, et al. Carga de Enfermedad atribuible al Tabaquismo en Chile. Buenos Aires, Argentina: Instituto de Efectividad Clínica y Sanitaria, 2014.

6.- MINSAL. Estudio de carga de enfermedad y carga atribuible en Chile. 2007.

7.- American Addiction Centers. Most addictive drugs. [30 agosto 2017]; Disponible en: http://americanaddictioncenters.org/adult-addiction-treatment-programs/mostaddictive/. 
8.- Instituto Nacional del Cáncer USA. Smokefreeespañol. [septiembre 2017]; Disponible en: https://espanol. smokefree.gov/.

9.- GUYATT G, OXMAN AD, AKL EA, KUNZ R, VIST G, BROZEK J, et al. GRADE guidelines: 1. Introduction-GRADE evidence profiles and summary of findings tables. J Clin Epidemiol. 2011; 64: 383-94. Epub 2011/01/05.

10.- BALSHEM H, HELFAND M, SCHUNEMANN HJ, OXMAN AD, KUNZ R, BROZEK J, et al. GRADE guidelines: 3. Rating the quality of evidence. J Clin Epidemiol. 2011; 64: 401-6. Epub 2011/01/07.

11.- HIGGINS DA. Assessing the risk of bias in included studies. Cochrane handbook for systematic reviews of interventions 5.0.1. Chichester, UK: John Wiley \& Sons; 2008.

12.- STEAD LF, BUITRAGO D, PRECIADO N, SANCHEZ G, HARTMANN-BOYCE J, LANCASTER T. Physician advice for smoking cessation. The Cochrane database of systematic reviews. 2013:CD000165. Epub 2013/06/04

13.- VIRTANEN SE, ZEEBARI Z, ROHYO I, GALANTI MR. Evaluation of a brief counseling for tobacco cessation in dental clinics among Swedish smokers and snus users. A cluster randomized controlled trial (the FRITT study). Prev Med [Internet]. 2015; 70:[26-32 pp.]. Disponible en: http://onlinelibrary.wiley.com/o/ cochrane/clcentral/articles/091/CN-01036091/frame. html.

14.- MCROBBIE H, BULLEN C, GLOVER M, WHITTAKER R, WALLACE-BELL M, FRASER T. New Zealand smoking cessation guidelines. $\mathrm{N} Z$ Z Med J. 2008; 121: 57-70.

15.- Guías Clínicas de Tratamiento de Personas con Depresión del MINSAL, 2006.

16.- ANTHENELLI RM, BENOWITZ NL, WEST R, ST AUBIN L, MCRAE T, LAWRENCE D, et al. Neuropsychiatric safety and efficacy of varenicline, bupropion, and nicotine patch in smokers with and without psychiatric disorders (EAGLES): a double-blind, randomised, placebo-controlled clinical trial. Lancet. 2016; 387(10037): 2507-20.

17.- WHITTAKER R, MCROBBIE H, BULLEN C, RODGERS A, GU Y. Mobile phone-based interventions for smoking cessation. The Cochrane database of systematic reviews. 2016;4:CD006611. Epub 2016/04/12.

18.- RUIZ M. El uso de las nuevas tecnologías en el tratamiento del tabaquismo. In: Editorial Aula Médica, editor. Tratado de tabaquismo. Madrid, España 2011. p. 387-94.

19.- Subsecretaría de telecomunicaciones. Informe Trimestral del Sector Telecomunicaciones - Primer Trimestre 2017. [31 agosto 2017]; Disponible en: http://www.subtel.gob.cl/estudios-y-estadisticas/informes-sectorialesanuales/.

20.- MUÑOZ RF, AGUILERA A, SCHUELLER SM, LEYKIN Y, PÉREZ-STABLE EJ. From online randomized controlled trials to participant preference studies: morphing the San Francisco Stop Smoking site into a worldwide smoking cessation resource. Journal of medical Internet research. 2012; 14: e64. Epub 2012/06/29

21.- STEAD LF, PERERA R, BULLEN C, MANT D, HARTMANN-BOYCE J, CAHILL $\mathrm{K}$, et al. Nicotine replacement therapy for smoking cessation. The Cochrane database of systematic reviews. 2012;11:CD000146. Epub 2012/11/16.

22.- BEHRAKIS P. Tobacco Cessation Guidelines for high risk populations The Health Programme of the European Union 2017.

23.- MILTON M MC, YEE S, BACKINGER C, MALARCHER A, HUSTEN C. Youth Tobacco Cessation: A Guide for Making Informed Decisions. Atlanta: U.S.: Centers for Disease Control and Prevention, 2004.

24.- LATIMER AE, KRISHNAN-SARIN S, CAVALLO DA, DUHIG A, SALOVEY P, O’MALLEY SA. Targeted Smoking Cessation Messages for Adolescents. J Adolesc Health. 50: 47-53.

25.- ZWAR N RR, BORLAND R, PETERS M, LITT J, BELL J. Supporting Smoking Cessation:a Guide for Health Professionals. Melbourne: The Royal Australian College of General Practitioners, 2011 (updated 2014).

26.- Ministry of Health. Recommendations of the New Zealand Guidelines for Helping People to Stop Smoking. New Zealand: 2014

27.- National Institute for Healthcare Excellence (NICE). Smoking: stopping in pregnancy and after childbirth. 2010.

28.- HUGHES JR, STEAD LF, HARTMANN-BOYCE J, CAHILL K, LANCASTER T. Antidepressants for smoking cessation. Cochrane Database Syst Rev. 2014;1:CD000031.

29.- CAHILL K, LINDSON-HAWLEY N, THOMAS KH, FANSHAWE TR, LANCASTER T. Nicotine receptor partial agonists for smoking cessation. The Cochrane database of systematic reviews. 2016:CD006103. Epub $2016 / 05 / 10$
Correspondencia a:

Dra. María Paz Corvalán B.

Sociedad Chilena de Enfermedades Respiratorias

Santa Magdalena 75, Oficina 701

Providencia

Santiago, Chile

Email: corvalanbarrosmariapaz@gmail.com 